\title{
EDITORIAL
}

\section{Marching to the beat of different drummers: individual airway response diversity}

\author{
R.H. Brown
}

The complicated structures of the lungs can be viewed in the most simplistic form as airways, vessels, and nerves. These structures work together to perform the marvellous feat of respiration and gas exchange. During disease states, one of these structures is impaired, either temporarily or permanently, and respiration and gas exchange are hindered. Asthma is a disease that primarily affects the airways. In asthma, while the aetiology may be varied, and remains a source of scientific controversy, the final common process is constriction of the airway smooth muscle, airway narrowing, and decreased ventilation that, in the severest form, can lead to death. The causes for this pathological constriction of the airways in asthmatics compared to healthy subjects are elusive and remain an area of intense research.

It is well recognised that the airway tree structure throughout the lungs is not uniform in structure or composition [1]. On the simplest level, the airways change in size from the trachea to the alveoli [2]. In addition, the cellular composition of the airways changes along the airway tree. For example, the amount of airway smooth muscle, cartilage, and nerves progressively decreases along the airway tree. Therefore, it is logical to suggest that the airways along the tree would not respond in a homogeneous manner in health or disease. However, the next step in the understanding of the heterogeneous response of the airways is not so intuitive.

Most investigators accept the idea of heterogeneity along the airway tree [3-5]. However, the idea of heterogeneous responses at the same level of the airway tree has not been clearly established. Recently, computer modelling has been used to simulate the process of bronchoconstriction, including airway heterogeneity. The amount and location of the constriction may be selectively adjusted and the resulting level of calculated lung resistance and elastance observed. LUTCHEN and GILLIS [6] found that both lung resistance and elastance were extremely sensitive to inhomogeneous constriction. A few highly constricted or nearly closed airways, randomly dispersed throughout the periphery of the lung, had a significant impact on the calculated breathing capacity. Of interest, their model was quite tolerant of very inhomogeneous changes in airway diameters, as long as there were no airways nearly or fully closed. THORPE and BATES [7] also evaluated inhomogeneous constriction in another computational model. They found similar results. The increases in lung resistance and elastance were largely due to the development of severe inhomogeneity in the airway tree that progressively isolates parts of the peripheral tissue from the central airways.

Imaging evidence for airway response heterogeneity has been present since the first report of the use of high-resolution computed tomography (HRCT) to measure airway reactivity in animals suggested that there was heterogeneity in the response of the airways that was unrelated to airway size [5].

Correspondence: R.H. Brown, Johns Hopkins School of Public Health, 615 North Wolfe Street, Baltimore, Maryland, 21205, USA. Fax: 1 4109550299. E-mail: rbrown2@jhem.jhmi.edu
Subsequent work from the same laboratory demonstrated that the route of administration of the agonist agent did not influence the airway heterogeneity [4]. The authors concluded that the observed airway response heterogeneity was an intrinsic function of the airways and not due to variability in the delivery of the agonist agent.

Other data that supports the notion of airway heterogeneity has been generated using other imaging modalities, such as magnetic resonance (MR) and positron emission tomography (PET) imaging. While MR and PET imaging are unable to demonstrate airway structures, they can, with the use of hyperpolarised helium for MR, demonstrate ventilation [8, 9], and with the use of ${ }^{13} \mathrm{~N}$-nitrogen for PET, demonstrate ventilation and perfusion inhomogeneities $[10,11]$. While quantification remains a daunting task, the images can provide qualitative insight into the extent of airway heterogeneity in humans. When viewing the images of the areas of the lungs that are not ventilated after methacholine challenge, the airway response heterogeneity is clear. There are large areas that are not ventilated, there are other areas that are partially ventilated, and there are areas that are completely ventilated. These images strongly suggest that there is a heterogeneous response of similar sized airways. To postulate otherwise, one would expect images of the lung after agonist challenge that would look like a bush that had been evenly pruned and thus the periphery removed. However, one sees erratic, inconsistent, large and small areas throughout the lung fields that are not ventilated, consistent with a heterogeneous response, even among the same size airways.

This heterogeneous nature of the response in similar size airways when measured by HRCT scans has frequently been seen as outside the limits of the technique resolution, i.e. "noise" rather than "signal". To reduce the "noise", data from individual airways of similar size are frequently either collapsed down to specific size stratum [3,4] or, at the extreme, to a single number [12], thus removing the unwanted "noise" of airway response and obscuring any true response heterogeneity.

We now have direct quantitative evidence for airway response heterogeneity. In the current issue of the journal, KING et al. [13] directly examine the issue of "signal" versus "noise" in airway response heterogeneity. They measured the responses of the airways in asthmatic and healthy subjects. Most important, they simultaneously measured the repeatability of their airway measurements. Using their technique to quantify the changes in size of the airways on the HRCT scans, they were not able to conclude that the small airways $(<2 \mathrm{~mm}$ in diameter) responded heterogeneously to the methacholine challenge, since the coefficient for the airway response in either the healthy or asthmatic subjects was not significantly different from the coefficient of repeatability of the measurement. However, they did find that in the airways $>2 \mathrm{~mm}$ in diameter, the airways of the asthmatic subjects showed a significant increase in the heterogeneity to the methacholine challenge. In contrast, in the healthy subjects, 
the coefficient for the airway response was not significantly different from the coefficient of repeatability of the measurement in the airways $>2 \mathrm{~mm}$ in diameter. The "noise" just became a "signal". The fact that the asthmatic subjects had a greater response heterogeneity in airways $>2 \mathrm{~mm}$ in diameter, compared with the healthy subjects, suggests that in asthmatic subjects it is not simply that they have an overall greater airway constrictor response, but that the response of their airways is also more variable.

These data, like much previous data, suggest a potential location for the pathological airway responsiveness in asthmatic subjects compared with healthy individuals. While limitations of the currently used HRCT techniques preclude definitive statements that the small airways in asthmatic subjects also respond in a heterogeneous manner compared with the airways in healthy individuals, future technical advances may show that the heterogeneous nature of airway responsiveness is not limited to the larger airways.

At first glance, the information from KING et al. [13] adds another layer of complexity to the already complicated nature of the airway responses in asthmatic and healthy individuals. However, on further examination, the significance of their findings may change the perspective of the aetiology of airway hyperresponsiveness. Consistent with computer modelling and qualitative imaging, it may be that it is not only the specific location of the heterogeneous responses, but the extent of the diversity of the airways and their response heterogeneity, both within the same size airways and among the different size airways, which may be a primary pathology of asthma. This revised focus on the heterogeneity of response may lead to a better understanding of why asthmatic subjects have symptomatic bronchoconstriction. With further technical advances in direct imaging and computer modelling, a clearer picture of how the pathophysiology of airway hyperresponsiveness leads to symptomatic asthma should be gained.

\section{References}

1. Von Hayek H. The Human Lung. New York and London, Hafner, 1960.
2. Weibel ER. Morphometry of the human lung. Heidelberg, Springer-Verlag, 1963

3. Okazawa M, Muller N, McNamara AE, Child S, Verburgt L, Pare PD. Human airway narrowing measured using high resolution computed tomography. Am J Respir Crit Care Med 1996; 154: 1557-1562.

4. Brown RH, Herold CJ, Hirshman CA, Zerhouni EA, Mitzner W. Individual airway constrictor response heterogeneity assessed by high resolution computed tomography. J Appl Physiol 1993; 74: 2615-2620.

5. Brown RH, Herold CJ, Hirshman CA, Zerhouni EA, Mitzner W. In vivo measurements of airway reactivity using high-resolution computed tomography. Am Rev Respir Dis 1991; 144: 208-212.

6. Lutchen KR, Gillis H. Relationship between heterogeneous changes in airway morphometry and lung resistance and elastance. J Appl Physiol 1997; 83: 1192-1201.

7. Thorpe CW, Bates JH. Effect of stochastic heterogeneity on lung impedance during acute bronchoconstriction: a model analysis. J Appl Physiol 1997; 82: 1616-1625.

8. Samee S, Altes T, Powers $\mathrm{P}$, et al. Imaging the lungs in asthmatic patients by using hyperpolarized helium-3 magnetic resonance: assessment of response to methacholine and exercise challenge. J Allergy Clin Immunol 2003; 111: 12051211.

9. Salerno M, Altes TA, Mugler JP 3rd, Nakatsu M, Hatabu H, de Lange EE. Hyperpolarized noble gas MR imaging of the lung: potential clinical applications. Eur J Radiol 2001; 40: 33-44.

10. Vidal Melo MF, Layfield D, Harris RS, et al. Quantification of regional ventilation-perfusion ratios with PET. $\mathrm{J} \mathrm{Nucl}$ Med 2003; 44: 1982-1991.

11. Tgavalekos NT, Venegas JG, Suki B, Lutchen KR. Relation between structure, function, and imaging in a threedimensional model of the lung. Ann Biomed Eng 2003; 31: 363-373.

12. Niimi A, Matsumoto $\mathrm{H}$, Amitani $\mathrm{R}$, et al. Airway wall thickness in asthma assessed by computed tomography. $\mathrm{Am}$ J Respir Crit Care Med 2000; 162: 1518-1523.

13. King GG, Carroll JD, Müller NL, et al. Heterogeneity of narrowing in normal and asthmatic airways measured by HRCT. Eur Respir J 2004; 24: 211-218. 